\title{
The dynamic characteristics of the mouse horizontal vestibulo-ocular and optokinetic response
}

\author{
A.M. van Alphen ${ }^{\mathrm{a}}$, J.S. Stahl ${ }^{\mathrm{b}}$, C.I. De Zeeuw ${ }^{\mathrm{a}, *}$ \\ ${ }^{a}$ Department of Anatomy, FGG, Erasmus University Rotterdam, P.O. Box 1738, 3000 DR Rotterdam, The Netherlands \\ ${ }^{\mathrm{b}}$ Department of Neurology, Case Western Reserve University, Cleveland, OH 44106, USA
}

Accepted 24 October 2000

\begin{abstract}
In the present study the optokinetic reflex, vestibulo-ocular reflex and their interaction were investigated in the mouse, using a modified subconjunctival search coil technique. Gain of the ocular response to sinusoidal optokinetic stimulation was relatively constant for peak velocities lower than $8^{\circ} \mathrm{s}$, ranging from 0.7 to 0.8 . Gain decreased proportionally to velocity for faster stimuli. The vestibulo-ocular reflex acted to produce a sinusoidal compensatory eye movement in response to sinusoidal stimuli. The phase of the eye movement with respect to head movement advanced as stimulus frequency decreased, the familiar signature of the torsion pendulum behavior of the semicircular canals. The first-order time constant of the vestibulo-ocular reflex, as measured from the eye velocity decay after a vestibular velocity step, was $660 \mathrm{~ms}$. The response of the vestibulo-ocular reflex changed with stimulus amplitude, having a higher gain and smaller phase lead when stimulus amplitude was increased. As a result of this nonlinear behavior, reflex gain correlated strongly with stimulus acceleration over the $0.1-1.6 \mathrm{~Hz}$ frequency range. When whole body rotation was performed in the light the optokinetic and vestibular system combined to generate nearly constant response gain (approximately 0.8 ) and phase (approximately $0^{\circ}$ ) over the tested frequency range of $0.1-1.6 \mathrm{~Hz}$. We conclude that the compensatory eye movements of the mouse are similar to those found in other afoveate mammals, but there are also significant differences, namely shorter apparent time constants of the angular VOR and stronger nonlinearities. (C) 2001 Elsevier Science B.V. All rights reserved.
\end{abstract}

Theme: Motor systems and sensorimotor integration

Topic: Oculomotor systems

Keywords: Mouse; Optokinetic reflex; Vestibulo-ocular reflex; Visually enhanced vestibulo-ocular reflex; Time constant

\section{Introduction}

In genetics studies, the mouse is probably the most important mammalian model, reflecting its technical advantages of short gestation, availability of embryonic stem cells that can be readily manipulated, and the large number of genetic probes already developed for use in this animal. Our knowledge of its ocular motor behavior, however, is still rudimentary, owing to the technical difficulty of recording eye movements in such a small animal. In the past eye movement recordings in mice have been attempted by electro-oculography [17], video oculography

\footnotetext{
*Corresponding author. Tel.: +31-10-408-7299; fax: +31-10-4089459 .

E-mail address: dezeeuw@anat.fgg.eur.nl (C.I. De Zeeuw).
}

$[2,20,24,25]$ or via the magnetic search coil technique, using temporary coils placed upon the eye at the time of the experiment $[10,11,22]$. The electro-oculography and older video oculography studies were flawed by calibration strategies based upon unrealistic assumptions that the gain of the optokinetic reflex (OKR) was unity at low stimulus velocities, while a more recent video study [20] failed to account for the anatomical parameters of the eye when geometrically converting measurements of pupil displacement to angular rotation of the eye (see Section 4). Within the past 2 years, the magnetic search coil technique of eye movement recording [29] has been adapted for use in the mouse in our laboratory $[10,11,22]$. The recordings in our previous studies were performed using a relatively large coil (3 $\mathrm{mm}$ in diameter) encircling the optical axis of the eye. While this technique was sufficient for the purposes of those studies, the relatively bulky coil may have distorted 
the normal ocular motor behavior. Indications of the potential problem were the low gain of the OKR [22] and the occurrence of a large phase lead during the vestibuloocular reflex (VOR) [10].

If valid quantitative comparisons between the ocular motor behavior of the mouse and larger species are to be made, a less invasive coil technique is necessary. We now introduce such a technique for the mouse, using a chronically implanted minicoil (1 $\mathrm{mm}$ in diameter). The new method confirms the qualitative similarities between compensatory eye movements in the mouse and those of larger afoveate mammals, but also suggests novel characteristics, including higher degrees of nonlinearity and unusually short time constants.

\section{Materials and methods}

All animal procedures described below were carried out under an animal care protocol that was approved by the local ethical committee of Erasmus University, Rotterdam.

\subsection{Animal care and surgical procedures}

Seventeen C57BL/6 mice were tested. The C57BL/6 strain is thought to perform well in behavioral tests and therefore the most commonly used strain for knockout and transgenic manipulations [14]. In order to assure alertness of the animals during experiments, the light-dark cycle of the animals was shifted by $12 \mathrm{~h}$.

Animals were anesthetized with a mixture of $\mathrm{O}_{2}, \mathrm{~N}_{2} \mathrm{O}$ and $2 \%$ halothane. An acrylic head fixation pedestal was formed and fixed to the skull by five screws (M1, $1.5 \mathrm{~mm}$ ). Screws were implanted on the frontal, parietal and interparietal bone plates. After completion of the construct, the head was positioned in a $70^{\circ}$ roll so the eye could be easily approached. A small incision was made in the conjunctiva on the temporal side of the eyeball. A pocket was bluntly dissected anterior to the insertion of the lateral rectus muscle. A copper wire coil (1 mm outside diameter, 60 turns, $1.0 \mathrm{mg}$ ) was placed in the pocket. The coil was fixed to the sclera with two sutures (10/0 nylon, Ethicon). The sutures were approximately aligned in the equatorial plane of the eye (Fig. 1). The conjunctiva was closed over the coil with an additional suture. The leads of the coil were carefully tunneled underneath the conjunctiva and the skin towards a miniature coaxial MMCX connector that was attached to the top of the acrylic head pedestal.

Full recovery from the surgery was not achieved until 5 days after implantation. In three mice the response to an optokinetic stimulus was monitored starting 3 days after implantation of the search coil. The OKR was elicited by a sinusoidal stimulus of $8 \%$ at $0.4 \mathrm{~Hz}$. Gain of the eye movement increased significantly from $0.63 \pm 0.07$ on the third to $0.76 \pm 0.06$ on the 6 th postoperative day $(P<0.05$, paired student $t$-test) (Fig. 2). Based on this recovery curve, only recording sessions from 6 days after coil
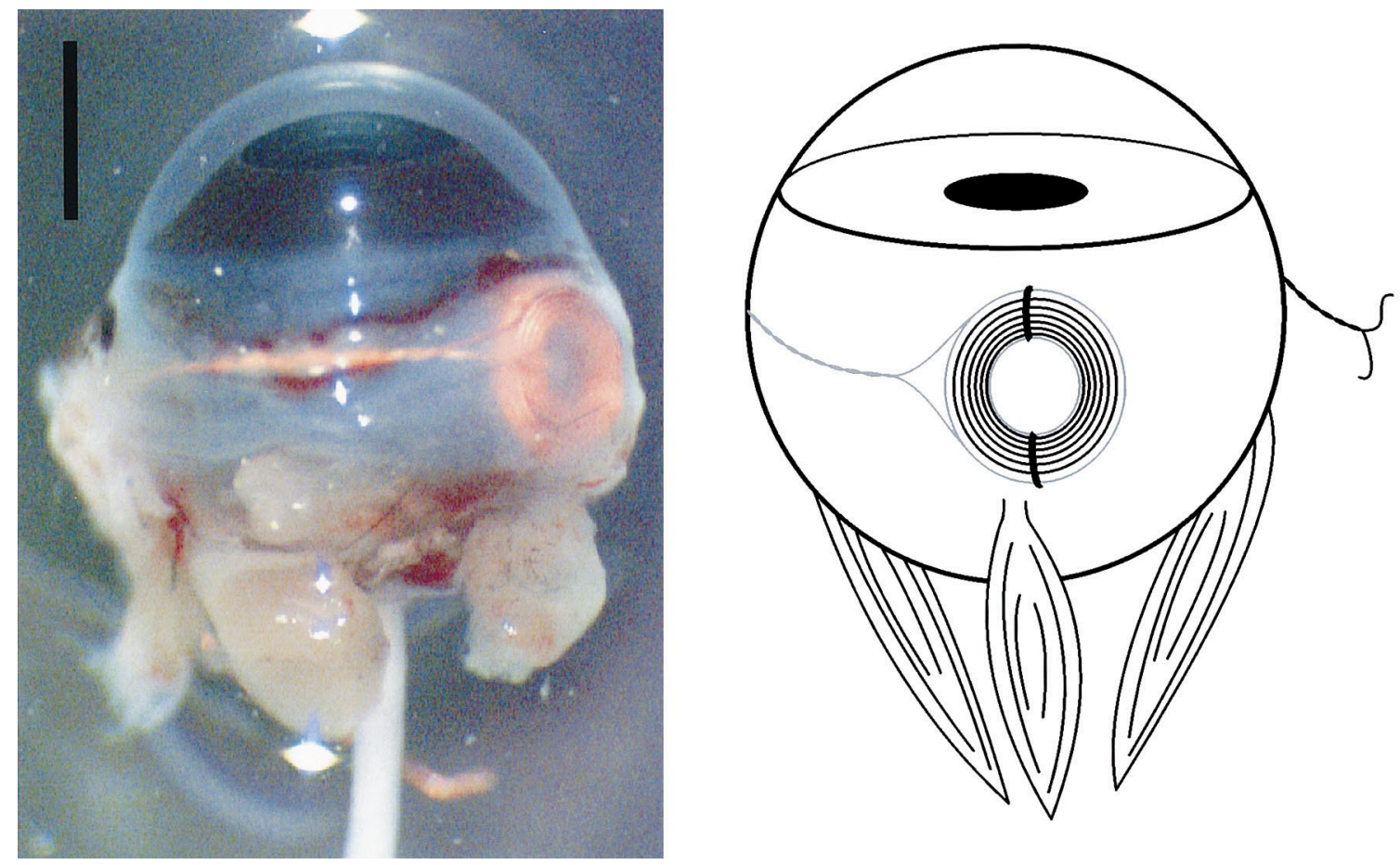

Fig. 1. Schematic drawing of the eye and a picture of a prepared mouse eye (ventral view). The scleral search coil is placed on the lateral surface of the left eye. The coil is attached to the sclera with two sutures. The conjunctiva is closed over the coil, so that both the coil and its lead are located underneath the conjunctiva. Scale bar $=1 \mathrm{~mm}$. 


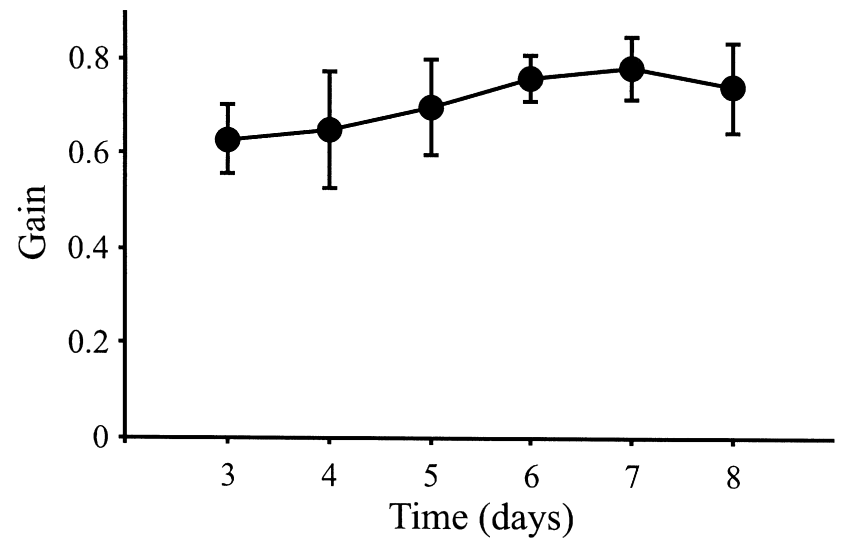

Fig. 2. The recovery from surgery was monitored in three animals. As a measure of recovery the response to an optokinetic stimulus of $0.4 \mathrm{~Hz}$ was taken ( $8^{\circ}$ s peak velocity). Gain of the response was averaged across animals and plotted against time, starting on the third day after implantation of the coil. The error bars show one standard deviation.

implantation were used in the analysis. In two cases it was necessary to include data that was recorded at postoperative day 5 , to construct a 3-day average for the animal.

\subsection{Eye movement recordings}

Mice were immobilized in a custom-made restrainer incorporating an aluminum plate to which the mouse's head fixation pedestal was bolted. The restraint assembly was then mounted within magnetic field coils (CNC Engineering, Seattle, WA) atop a vertical-axis turntable (Biomedical Engineering Co., Thornwood, NY). The midpoint of the interaural line was placed in the center of rotation for the optokinetic drum and the turntable. In order to maximize linearity of the eye movement transduction, the field coils were oriented about the mouse so that the horizontal magnetic field lines were approximately parallel to the surface of the eye-coil. Calibration was then achieved by rotating the field coils over $\pm 10^{\circ}$ about this optimum position while the animal maintained a stationary eye position. The amplifiers were adjusted to remove any DC offset. Thus, zero eye position corresponded to the animal's eye position at the time of calibration, and our recordings reflect eye position with respect to this resting position. Since our recordings (see below) suggest that the mouse, like the rabbit [7], tends to restrict eye eccentricity to a relatively limited range, this arbitrary zero position is probably no more than a few degrees from the center of the animal's preferred ocular motor range.

Optokinetic stimuli were delivered with a striped drum (width of bars was $4^{\circ}$ ). The drum had a diameter of $26 \mathrm{~cm}$ and enclosed the animal from zenith to $45^{\circ}$ below the horizon. To test the performance of the optokinetic system two different approaches were used. First, the frequency response of the OKR was tested by rotating the drum at frequencies of $0.1,0.2,0.4,0.8$ and $1.6 \mathrm{~Hz}$ with a peak velocity of $8^{\circ} / \mathrm{s}$. The effect of peak velocity on gain of the
OKR was evaluated by using sinusoidal stimuli at $0.4 \mathrm{~Hz}$ and peak velocities of $2,4,8,16$, and $32^{\circ}$ s. In addition, the drum was rotated at a constant velocity to elicit an optokinetic nystagmus. The optokinetic nystagmus was measured at the same stimulus velocities as the sinusoidal OKR. Velocity step stimuli $\left(6^{\circ} / \mathrm{s}\right)$ were also delivered, by randomly alternating -3 and $3 \%$ s constant velocity rotation.

The VOR was evaluated during sinusoidal, whole body rotation in the dark. Sinusoidal stimulation was performed at $0.1,0.2,0.4,0.8$, and $1.6 \mathrm{~Hz}$ and stimulus amplitudes of 5 or $10^{\circ} / \mathrm{s}$. Velocity steps were delivered by alternating table velocity from -15 to $15^{\circ} / \mathrm{s}$ at a rate of $0.1 \mathrm{~Hz}$. Peak acceleration of the turntable during a velocity step was $400^{\circ} / \mathrm{s}^{2}$. The visually enhanced vestibulo-ocular reflex (VVOR) was tested using the VOR stimulus parameters, while the animal viewed the illuminated, earth-fixed drum. The OKR, VOR and VVOR in response to sinusoidal stimuli were tested in six mice. The stimuli were delivered in three sessions on consecutive days. Each session consisted of three trials per stimulus. OKR, VOR and VVOR trials were randomly alternated within the session. Optokinetic and head velocity step stimuli were delivered to 11 and 10 animals, respectively.

\subsection{Data analysis}

All recorded signals were digitized at $500 \mathrm{~Hz}$ with 32-bit precision (CED, Cambridge, UK). Eye, head and drum position were differentiated off-line, using a threepoint software algorithm. Sinusoidal data was digitally filtered at a frequency of $20 \mathrm{~Hz}$ using a software Butterworth filter. Fast phases were identified and excised from the eye velocity data using combined velocity and acceleration criteria. The gain and phase of the response were calculated from the remaining slow-phase portions of the velocity signal by fitting a sine wave using least-square optimization. In this analysis the fundamental frequency of the eye movement was assumed to equal the stimulus frequency. Gain and phase values were combined per trial to yield session averages. Session averages were again averaged to yield final gain and phase values per mouse. All values reported are mean \pm S.D. weighting each mouse equally.

The eye movement recordings obtained after optokinetic stimulation with a constant velocity were treated in the same way as sinusoidal traces. After fast phases were excised from the time base, steady-state eye velocity was measured as the average slow phase eye velocity. Average slow phase eye velocity was calculated across all remaining samples spanning a time period from $10 \mathrm{~s}$ after the onset of eye movement to the moment the light was extinguished.

The latency of the optokinetic response to a velocity step was determined by measuring the onset of change in eye velocity in response to a velocity step of the drum. 
After data was differentiated it was filtered at $60 \mathrm{~Hz}$. At least 35 step responses in each direction were averaged, aligned upon the onset of stimulus transition. We excluded any trials in which a fast phase occurred within $1 \mathrm{~s}$ before or after the stimulus transient. The averaged velocity curves for clockwise and counterclockwise stimuli were each referenced to the mean pre-step eye velocity and then superimposed. We defined the onset of the response to the velocity step as the last point of intersection between the re-referenced averaged eye velocity curves. Latency was measured as the difference between this point of divergence and the onset of drum movement.

We determined the dominant time constant of the VOR from the averaged responses to at least 20 velocity steps of the turntable. Eye velocity $\left(E^{\prime}(t)\right)$ was again obtained by differentiation of eye position traces and filtered at $60 \mathrm{~Hz}$. We fitted a single exponential curve $\left(E^{\prime}(t)=A^{*} \mathrm{e}^{(-t / \tau)}+B\right)$ by least-square optimization to the epoch spanning $0.2-1.2$ $\mathrm{s}$ after the reversal of turntable direction. In this equation $\tau$ was taken as the time constant for the VOR.

All statistical analyses were performed using a commercially available package (Prism, GraphPad Software, San Diego, CA).

\section{Results}

\subsection{General findings}

At the beginning of each recording session mice were allowed to habituate to the restrainer for $15 \mathrm{~min}$. During this period eye position was recorded. Spontaneous gazeshifting eye movements (saccades) rarely occurred, and when they did, they were small. Thus, eye-in-orbit positions generally remained within $\pm 7^{\circ}$ of the arbitrary zero position defined during calibration (see Section 2). The rarity of saccades might indicate that mice, like other afoveate mammals, normally use combined eye-head saccades to explore their environment [13]. When spontaneous saccades were made, the new eye position was never held very long before the eye was returned toward the central position by a centripetal saccade or a smooth drift. In order to determine if the eye drift was due to leakage of the velocity-to-position (neural) integrator, gaze holding ability was evaluated in ten mice. In these mice the eye was brought to an eccentric position by slowly moving the turntable in the illuminated environment. After a new position in the orbit was reached the light was turned off. The decay of eye position $(E(t))$ back to resting position was measured by fitting a single exponential $(E(t)=$ $\left.A^{*} \mathrm{e}^{(-t / \tau)}+B\right)$ and the time constant $(\tau)$ of this decay was determined from the fit (Fig. 3). This procedure yielded an average time constant of $2.1 \pm 0.7 \mathrm{~s}$ for the neural integrator. The maximal eye deviation that could be reached in this manner ranged $5-9^{\circ}$ and is reflected by the sum of $A$ and $B$ in the equation above. Beyond this range, centripetal

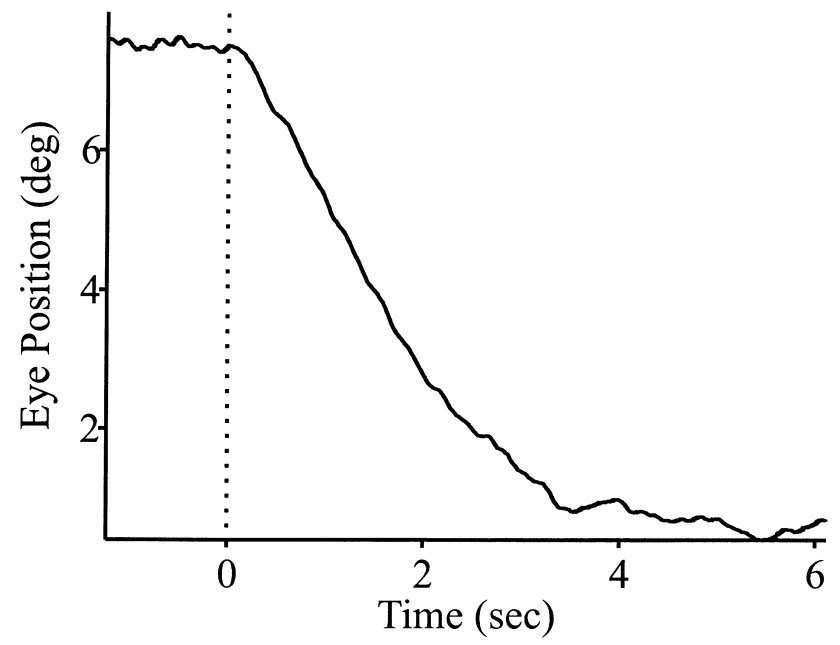

Fig. 3. An example of the gaze-holding ability of the mouse in the dark. The eye was brought to an eccentric position by slowly rotating the animal in the light. The consequent VVOR resulted in a displacement of the eye approximately $7^{\circ}$ from the rest position. At the dotted line the light was extinguished. The eye decayed back to its resting position with an approximately exponential time course.

fast phases occurred. There was little variation in the final resting position ( $B$ in the equation above) between trials. Averaged across animals, the standard deviation of $B$ was only $1^{\circ}$, confirming that the arbitrary zero position as defined was very close to the true neutral eye position, and demonstrating how narrow the range of preferred resting positions was.

\subsection{Optokinetic responses}

When the optokinetic drum was rotated sinusoidally, eye movement responses were virtually sinusoidal, indicating the linear behavior of the optokinetic response (OKR) at low stimulus velocities. Fig. 4A shows a representative response to a stimulus of $0.2 \mathrm{~Hz}, 8 \% \mathrm{~s}$ peak velocity $\left(6.3^{\circ} / \mathrm{s}\right.$ amplitude). At higher peak-velocities responses tended to become distorted, evincing a more triangular profile. Such responses have been described for other afoveate animals $[6,21]$ and reflect velocity saturation in the optokinetic system. Sinusoids were fit to the data to determine the gain and phase at the fundamental frequency of the response. In each animal the gain and phase of the eye movement were averaged across three recording sessions. The average standard deviation of the gain in these three sessions ranged from 0.03 to 0.07 , showing that measurements within each session were in good agreement. Average gain of OKR in the mouse was constant at $0.77 \pm 0.02$ for stimulus velocities of $8^{\circ} / \mathrm{s}$ and smaller, but decreased at higher velocities (Fig. 4B).

Because of the obvious velocity dependence of the OKR, the frequency response of the OKR was evaluated at a constant velocity of $8^{\circ} / \mathrm{s}$. Gain was $0.70 \pm 0.07$ at $0.1 \mathrm{~Hz}$, $0.74 \pm 0.06$ at $0.8 \mathrm{~Hz}$, and $0.57 \pm 0.07$ at $1.6 \mathrm{~Hz}$ (Fig. 4C). 

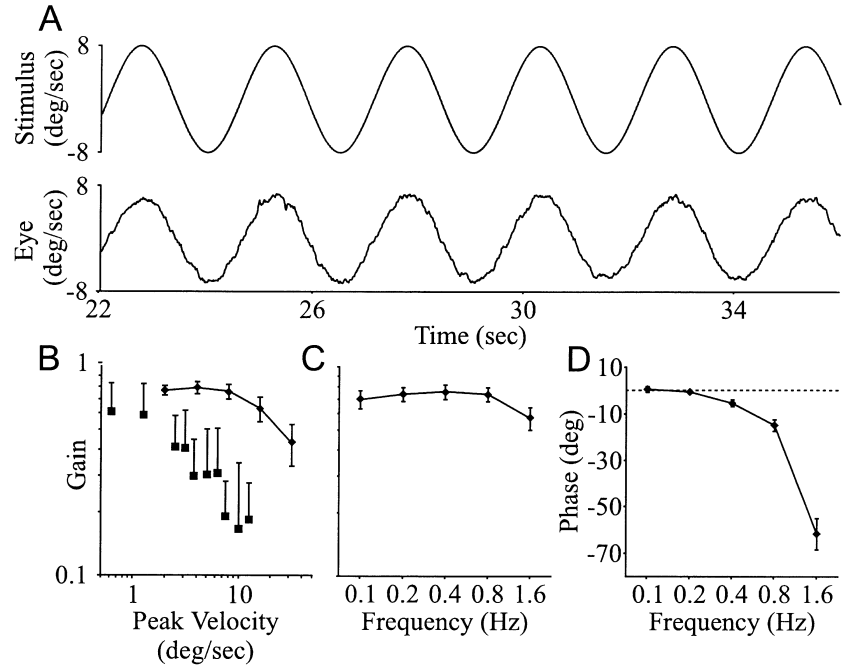

Fig. 4. (A) An example of a response to a sinusoidal optokinetic stimulus at $0.2 \mathrm{~Hz}$ and peak velocity $8^{\circ} \% \mathrm{~s}$. (B) Average gain of six animals, of the OKR to $0.4 \mathrm{~Hz}$ sinusoidal stimuli plotted against peak velocity of the stimulus $(\downarrow)$. Error bars indicate one standard deviation. Gain of the response remained constant around 0.8 for velocities lower than $8 \%$ s. For comparison, earlier data obtained using the large coil (diameter: $3 \mathrm{~mm}$ ), is also plotted (ם). (C) Average gain of the OKR to $8 \%$ s (peak velocity) sinusoidal stimuli, plotted against the frequency of the stimulus. (D) Phase of eye velocity relative to drum velocity, plotted against stimulus frequency $\left(8^{\circ} / \mathrm{s}\right.$ peak velocity). A negative value indicates a phase lag with respect to the stimulus.

Eye velocity progressively lagged drum velocity as stimulus frequency increased. At $0.1 \mathrm{~Hz}$, eye velocity lagged drum velocity by $0.5 \pm 1.3^{\circ} / \mathrm{s}$; at $1.6 \mathrm{~Hz}$ the lag had increased to $61.8 \pm 6.7^{\circ} / \mathrm{s}$ (Fig. 4D).
The phase of the optokinetic response reflects a combination of the system's fixed delay and dynamic terms. In order to distinguish these two components, we determined the latency of the optokinetic pathway by averaging the eye velocity response to random velocity steps (Fig. 5). The system delay measured $70 \pm 12 \mathrm{~ms}$.

The OKR was also evaluated during constant velocity stimulation. The response to sudden illumination of the rotating drum consisted of a rapid initial rise in eye velocity followed by a slow build-up to a steady state (Fig. 6A). The size of the initial rise in slow-phase eye velocity was dependent on stimulus velocity (Fig. 6B). A single exponential could be fitted to the data, showing that eye velocity saturated at $9.8^{\circ} \%$ for higher stimulus velocities $\left(r^{2}=0.98\right)$. Saturation of initial eye velocity is in reasonable agreement with the results from sinusoidal stimulation, which indicated that steady-state sinusoidal gain saturates at peak stimulus velocities of approximately $8^{\circ} / \mathrm{s}$. The steady-state constant velocity gain ranged from $0.75 \pm 0.10$ at $2 \%$ so $0.71 \pm 0.02$ at $32^{\circ} / \mathrm{s}$ (Fig. 6C).

We attempted to elicit optokinetic after nystagmus (OKAN) by extinguishing the lights at least $35 \mathrm{~s}$ after the onset of eye movement. Under our conditions, a classic sustained OKAN was rarely observed. Following a $32 \%$ stimulus, one or two beats of OKAN occasionally occurred, but the duration of this nystagmus was never long enough to allow a time constant to be measured. The lack of OKAN, the frequent departure of the slow buildup from an exponential profile, and the relatively large phase leads of VOR at low stimulus frequencies (see below) all suggest that velocity storage in the mouse is minimal under our stimulus conditions.

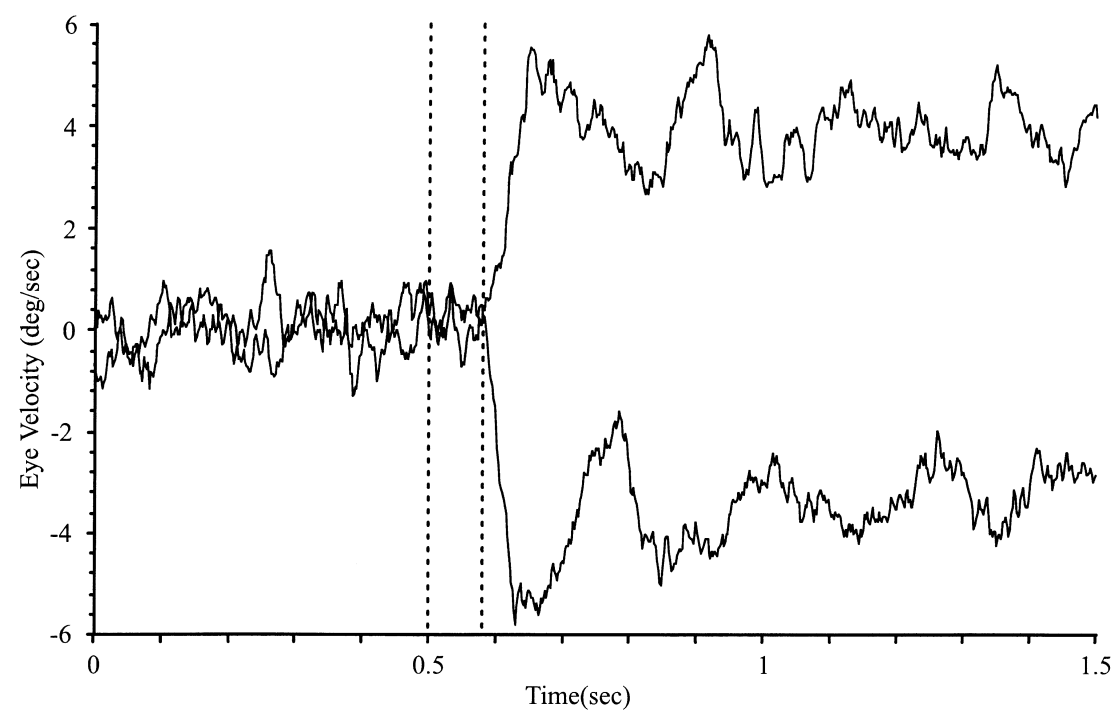

Fig. 5. Average of 61 responses to a $6 \%$ s $(-3 \%$ to $3 \%$ s) velocity step of the drum. The upper trace shows the average response for a step in clockwise direction, while the lower trace shows the response to a step in counter-clockwise direction. The eye velocity traces were shifted so that the average eye velocity was $0 \%$ s over the $500 \mathrm{~ms}$ prior to the onset of stimulus turn around. The first dotted line indicates the onset of stimulus turn around. The second doted line indicates the last point of intersection between the traces of clockwise and counterclockwise eye movement. The distance between the two points was taken as the latency of the optokinetic response ( $77 \mathrm{~ms}$ in the graph). 

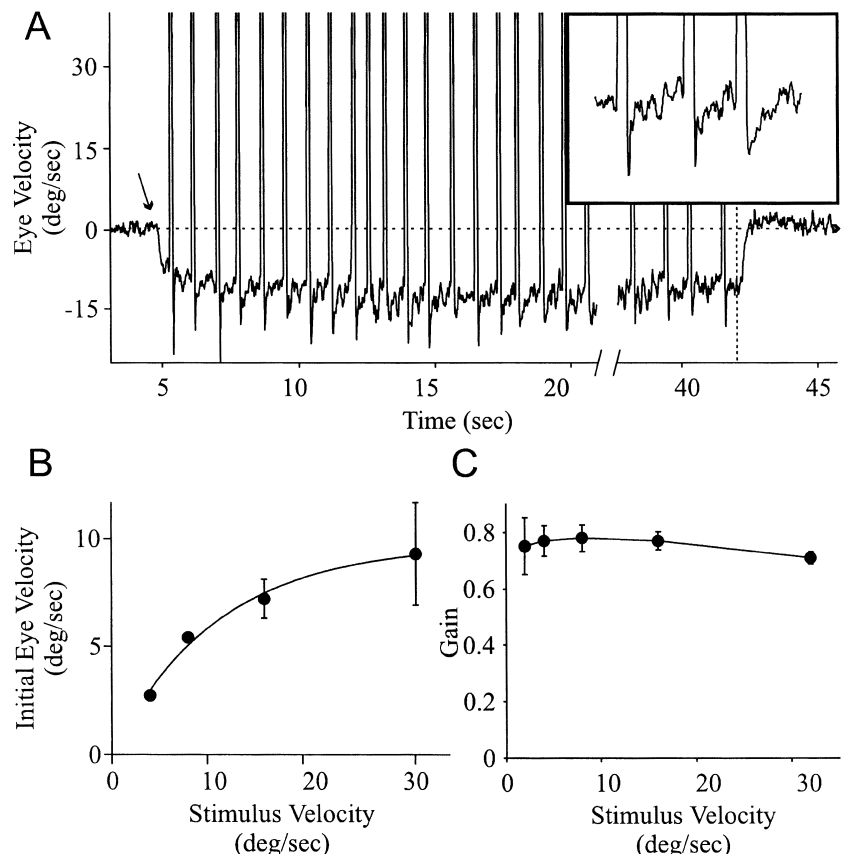

C

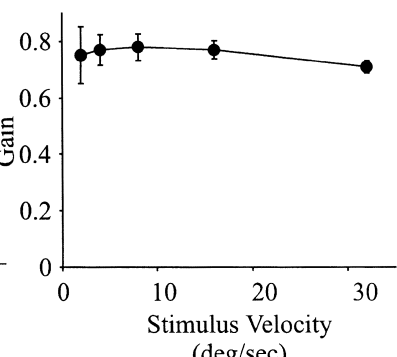

Fig. 6. (A) An example of the OKR to a constant $16^{\circ} / \mathrm{s}$ leftward rotation of the drum. After light was turned on (arrow), a quick initial rise in eye velocity occurred, which was followed by a gradual increase in slow phase eye velocity until a steady state was reached. Steady-state eye velocity was maintained for at least $15 \mathrm{~s}$, after which the light was turned off (vertical dotted line). Hardly any OKAN was observed. The inset shows a magnification of three beats of optokinetic nystagmus. Note the decrease of eye velocity during each slow phase. (B) The amplitude of the quick initial rise in eye velocity was plotted against stimulus velocity. An exponential was fitted to the data (solid line). The fit revealed a saturation of initial eye velocity for higher stimulus velocities at $9.8^{\circ} / \mathrm{s}$. (C) The steady-state gain of the OKR in response to constant velocity stimulation was plotted against stimulus velocity. Error bars show one standard deviation.

\subsection{Vestibular responses}

Horizontal VOR was elicited by whole body rotation of the animal in the dark. Unlike the OKR, which became distorted at high stimulus frequencies/velocities, vestibular eye movements were uniformly sinusoidal for all stimulus parameters we employed. At low frequencies $(0.1 \mathrm{~Hz})$ no response could be obtained and gain was consequently assumed to be 0 . While this finding could indicate a threshold effect, it is also possible that a response was present but could not be detected in the system noise (which measured $0.2^{\circ}$ peak-to-peak with the animal at rest). In all animals a systematic effect of stimulus amplitude on gain and phase was seen. Therefore, average values are reported separately for different stimulus amplitudes.

For all amplitudes the VOR showed properties of a high-pass filter in that gain increased and phase lead decreased as frequency was increased (Fig. 7A,B). For stimulus amplitudes of $10^{\circ}$, gain rose from $0 \pm 0$ to $0.57 \pm 0.08$ as frequency was increased from 0.1 to $1.6 \mathrm{~Hz}$.
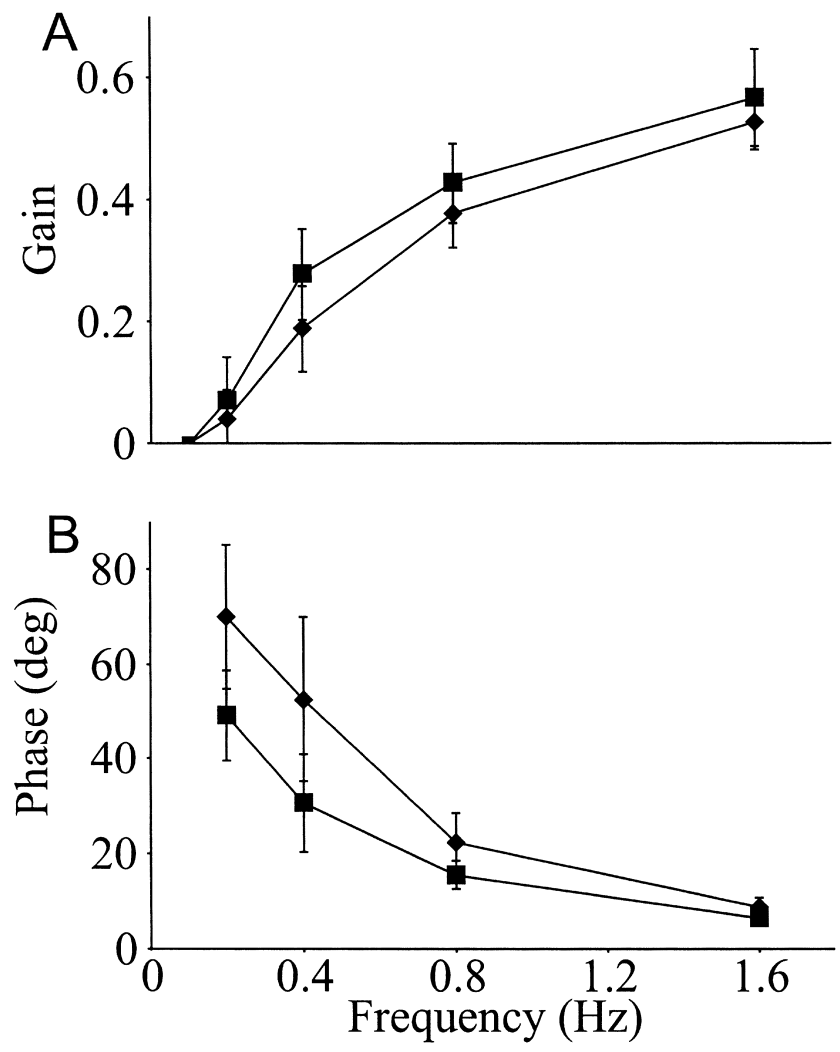

Fig. 7. Gain (A) and phase (B) of the VOR were plotted against frequency for $5^{\circ}(\diamond)$ and $10^{\circ}(\boldsymbol{\square})$ amplitude. Positive phase values indicate a phase lead of eye velocity with respect to head velocity. No response could be detected at $0.1 \mathrm{~Hz}$ for either amplitude. Note the dependence of both VOR gain and phase upon stimulus amplitude.

At $5^{\circ}$ amplitude the curve reached its maximum value of $0.53 \pm 0.05$ at $1.6 \mathrm{~Hz}$. Eye movement led head movement at all tested frequencies and amplitudes. Phase lead decreased with increasing frequency, from $49.0 \pm 9.5^{\circ}$ at $0.2 \mathrm{~Hz}$ to $6.4 \pm 1.4^{\circ}$ at $1.6 \mathrm{~Hz}\left(10^{\circ}\right.$ stimulus amplitude $)$.

In the range of tested frequencies, the amplitude of the stimulus also affected gain and phase (Fig. 7A,B). A two-way analysis of variance was performed on the average gain and phase data, using frequency and amplitude as independent variables. The effect of amplitude on both gain and phase proved to be significant $(P=0.0065$ and $P<0.0001$, respectively).

Under the assumption that the low frequency behavior of the average VOR phase curve was determined by first order high-pass characteristics, the data was fitted with a single-pole transfer function (Phase $=\operatorname{atan}\left(\omega^{-1} \tau^{-1}\right) *(180 /$ $\pi)$ ). This calculation yielded values of $370 \pm 40$ and $690 \pm 50 \mathrm{~ms}$ for the VOR time constant at $5^{\circ}$ and $10^{\circ}$ amplitudes. The correlation coefficients for the fits to the phase curves were 0.77 and 0.85 , respectively. However, even at $10^{\circ}$ the calculated time constant is still exceptionally low by comparison to other animals [3,28,31]. Therefore, we validated the value by measuring it in another fashion - by analyzing the eye velocity response to a 

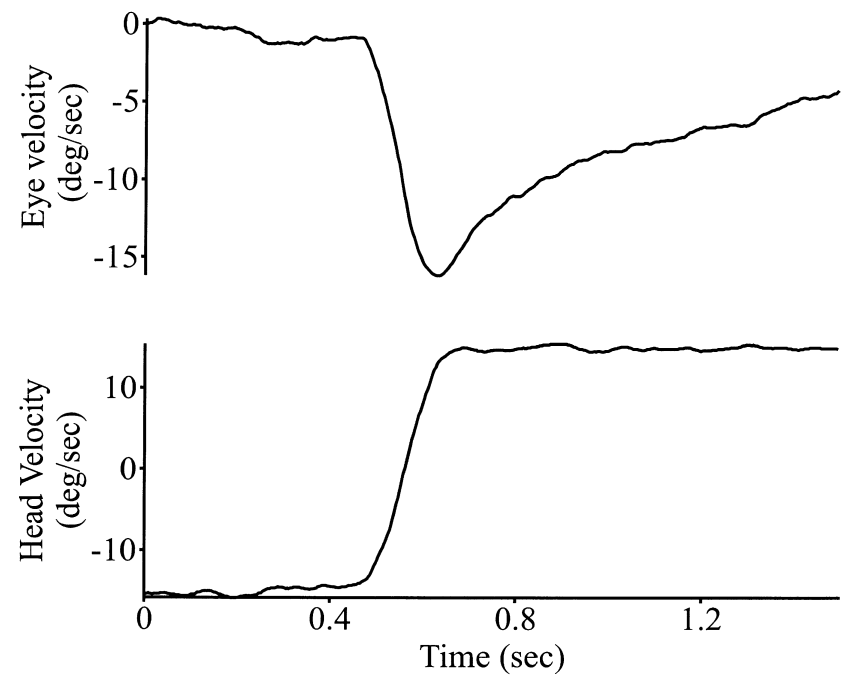

Fig. 8. An average of 20 eye velocity responses (upper trace) to a velocity step of the turntable (lower trace) was constructed. The table changed direction of rotation from $-15^{\circ} / \mathrm{s}$ to $15^{\circ} / \mathrm{s}$ at $0.1 \mathrm{~Hz}$ (peak acceleration of $400^{\circ} / \mathrm{s}^{2}$ ). Eye velocity decayed back to $0^{\circ} / \mathrm{s}$ with an approximately exponential time course. Note the absence of eye movement in the period before the step, when the eye has fully decayed to $0^{\circ} / \mathrm{s}$.
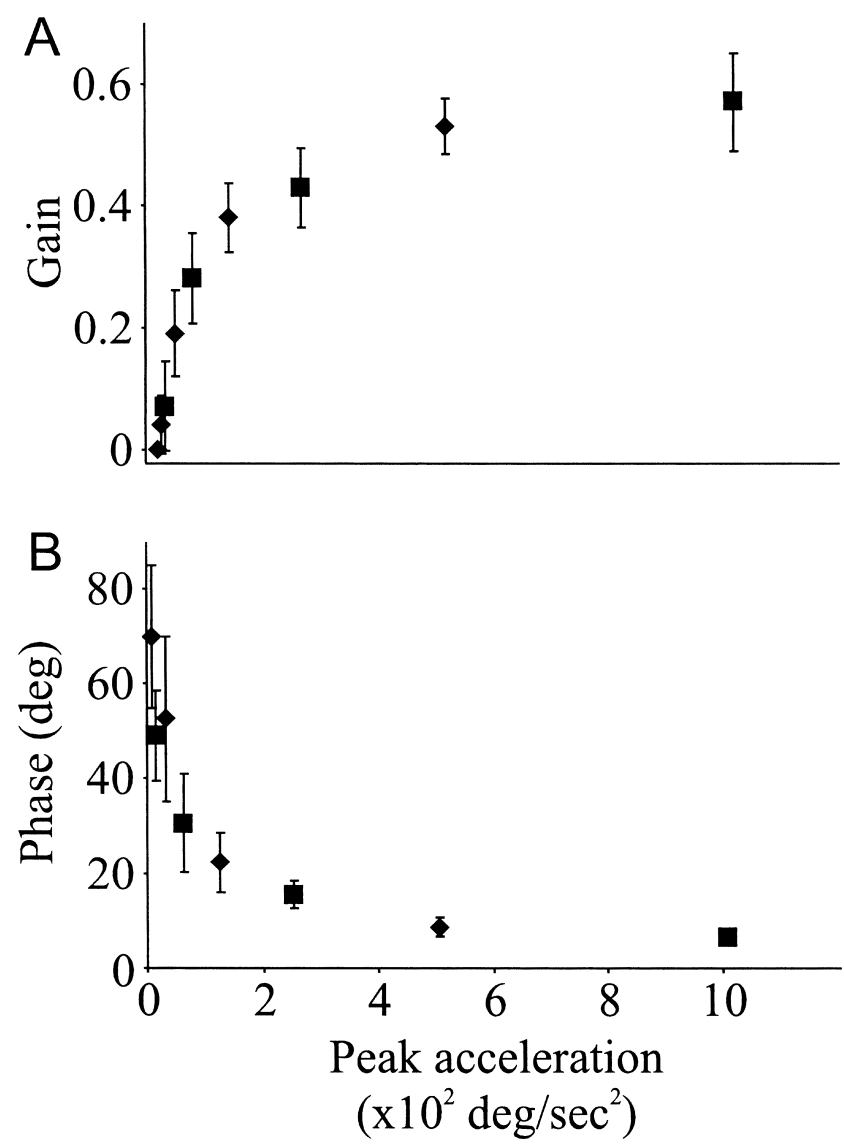

Fig. 9. Gain (A) and phase (B) of the VOR were plotted against peak acceleration of the head. Symbols depict $5^{\circ}(\downarrow)$ and $10^{\circ}(\mathbf{\square})$ amplitude. The results from all stimulus amplitudes lie upon a single curve. vestibular velocity step in the dark. After a step of $30^{\circ} / \mathrm{s}$, eye velocity decayed with an approximately exponential time course. A single exponential was fitted to obtain the time constant of the decay (Fig. 8). The average time constant was thus estimated to be $660 \pm 280 \mathrm{~ms}$.

The influence of amplitude on both phase and gain of the VOR reflects a nonlinearity in the vestibular system of the mouse. In fact, plotting gain and phase against peak acceleration instead of frequency collapsed the family of curves shown in Fig. 7 to a single curve (Fig. 9A,B). If the VOR was solely dependent on frequency, then separate curves should emerge in such a plot, for each set of frequencies. As the figure shows this separation does not occur and therefore the most important factor that determines the gain in the studied frequency range appears to be peak acceleration.

\subsection{Visuo-vestibular interaction}

VVOR gain was $0.78 \pm 0.02$ over the entire frequency range tested. Amplitude of the stimulus signal had no influence on gain (Fig. 10A). Phase of the eye movement was close to zero at all stimuli (Fig. 10B). Phase lead decreased slightly when frequency was increased, changing to a moderate phase lag at $1.6 \mathrm{~Hz}$.

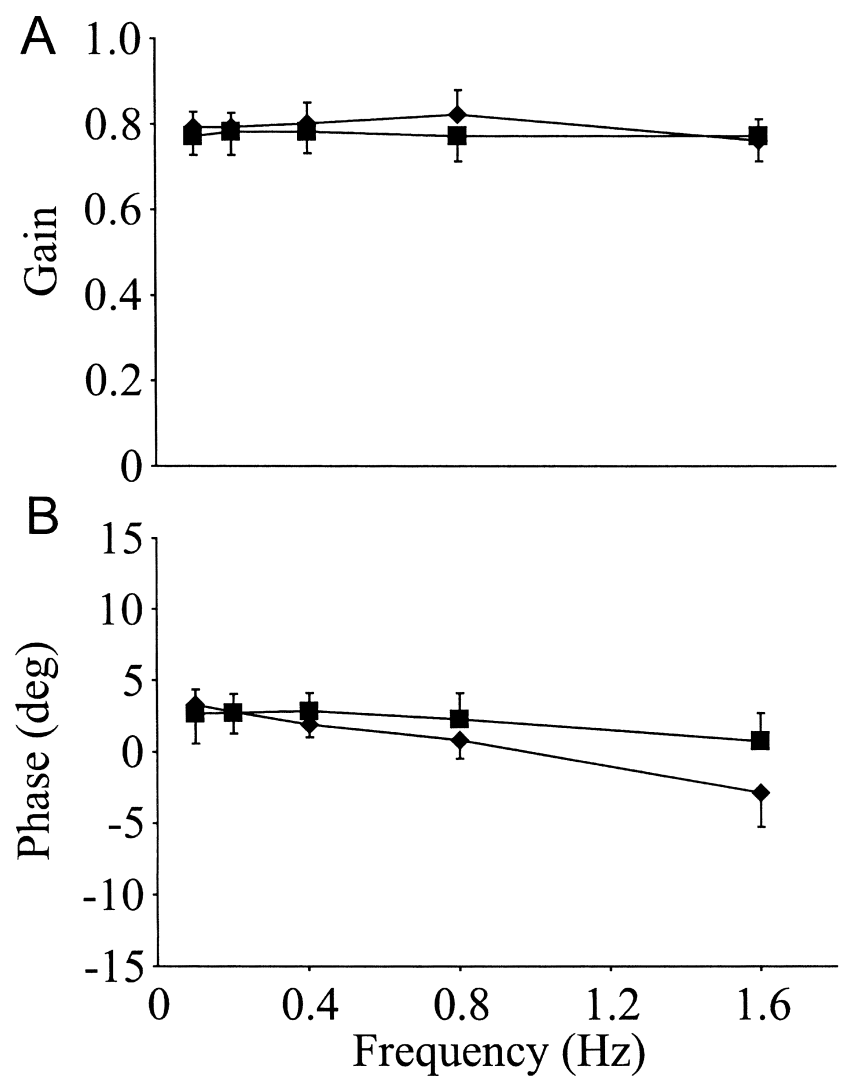

Fig. 10. Average gain (A) and phase (B) of the VVOR were plotted against frequency for $5^{\circ}()$ and $10^{\circ}(\mathbf{\square})$ amplitude. These indices of VVOR performance were roughly constant across the entire tested frequency range. 


\section{Discussion}

The present experiments have yielded a detailed description of compensatory eye movements in the mouse. In many respects optokinetic and vestibular eye movements in this species were similar to those of other afoveate species, such as the rabbit. Eye movements could be elicited by rotation of a surrounding striped drum or by whole body rotation. The horizontal frequency response of the optokinetic system was found to be essentially low pass, in that its gain fell and phase lag increased as frequency increased. Similar characteristics have been shown for the OKR in numerous species $[6,16,18,27]$. Gain at low peak velocities was relatively constant in the mouse (range 0.7-0.8), and comparable to values reported for the rabbit $(0.7-0.8)[6,12,26]$ and the rat $(0.7-0.9)$ [18]. When peak stimulus velocity was increased, gain of the response declined proportionally. The form of the OKR velocity-tuning curve reflects a saturation occurring in the visual pathways above stimulus velocities of $8-9^{\circ} / \mathrm{s}$ in the closed-loop condition. In the cat this value has been reported to be $6 \%$ s [16] and in the rabbit $1 \%$ s [6].

The response of the mouse to constant velocity optokinetic stimuli exhibited the familiar biphasic profile seen in other species [5,6,18,23,27]. An initial fast rise in eye velocity was followed by a slower build-up to a final steady state. In foveate species the initial acceleration is very prominent. It has a gain between 0.55 and 0.65 for stimuli of $30^{\circ} / \mathrm{s}$ and higher in monkeys [5] and is thought to reflect in large part the cortically mediated smooth pursuit system. In afoveate species, which lack smooth pursuit, the initial component is smaller and is thought to reflect more direct subcortical pathways, perhaps involving the accessory optic system. The magnitude of the initial response in the mouse $\left(9^{\circ} / \mathrm{s}\right)$ was comparable to other afoveate species, in which initial OKR eye velocities around $10^{\circ} \%$ are reported $[6,18,21]$.

\subsection{The neural integrator is less prominent in the mouse}

The velocity-to-position (neural) integrator generates a desired eye position command from the velocity-encoded premotor signals [15,32]. A number of lines of evidence suggest that the neural integrator is comparatively weak in mice. First, fast phases of vestibular and optokinetic nystagmus were often followed by backwards drifts. These decays, which are consistent with reduced neural integration, became more pronounced at lower stimulus velocities when fewer fast phases occurred. Second, direct tests of eye stability following step-, vestibularly-evoked displacements revealed an integrator time constant of only $2.1 \pm 0.7$ $\mathrm{s}$. This value is very low compared to $20 \mathrm{~s}$ in cat [30] and monkey [4]. Third, we measured a transport delay of 70 $\mathrm{ms}$ for the mouse optokinetic system. OKR phase in lateral eyed mammals has been said to be dictated by this delay in the optokinetic system $[6,18]$. However, at stimulus frequencies below $1.0 \mathrm{~Hz}$, a $70 \mathrm{~ms}$ delay would cause a larger phase lag than was actually observed. This result would be explained by the weaker neural integrator which, by producing less than the usual amount of phase lag, would simulate the presence of a lead element, and so counter the phase lag produced by the visual system's pure delay element. Of note, at frequencies above $1.0 \mathrm{~Hz}$, phase lag became larger than the value predicted by a fixed delay. This result may reflect additional low-pass dynamic characteristics in the optokinetic system, that become dominant at higher frequencies where the lead-producing effects of the leaky neural integrator fall off. Fourth and finally, the short time constant of the VOR provides additional evidence for a comparatively weak neural integrator.

VOR dynamics have been generally explained in terms of the mechanical properties of the peripheral organ [34], which is modeled as an overdamped torsion pendulum. In the absence of velocity storage (which we find to be weak in the mouse, see below) the time constant of the VOR should mirror that of the cupula [32]. Based upon allometric considerations, that cupular time constant should be approximately $3 \mathrm{~s}$ in the mouse [19]. Primary vestibular afferents have not been recorded in the mouse, but in the rat their time constant measures $3.4 \mathrm{~s}$ [9]. The mouse VOR has the expected high pass characteristics, i.e. gain increases and phase lead decreases with increasing frequency. However, we calculated the mouse VOR time constant from both frequency- and time-domain methods to be around $690 \mathrm{~ms}$, well below the probable cupular time constant. This low time constant is also consistent with the relative phase-leading effects of a weak velocity to position integration.

The integrator time constant could be affected by the presence of a scleral search coil. Placement of a search coil may change the viscoelastic properties of the oculomotor plant in a way that would mimic a reduced time constant for the neural integrator. However, such an effect of the minicoil cannot fully account for the behavior of the mouse oculomotor system since recent experiments, using video oculography, confirm the finding that the neural integrator has an unusual short time constant in the mouse (Stahl, personal communication).

\subsection{Velocity storage is impaired in the mouse}

The velocity storage mechanism serves to store velocity of the visual surround to stabilize gaze during constant velocity motion of the world [5]. When a constant velocity optokinetic stimulus is presented eye velocity typically exhibits a delayed, exponential increase towards a steady state. This build-up reflects charging of the velocity storage mechanism. In the mouse build-up of eye velocity differed from that described in other species. It was smaller and had a more variable profile. Thus velocity storage 
appears to be weak in the mouse, at least under the current stimulus conditions. In support of this conclusion, extinction of the lights following prolonged unidirectional optokinetic stimulation never elicited a well developed, optokinetic after nystagmus (OKAN).

\subsection{Non-linear aspects of the VOR in the mouse}

Previous studies have focused on the degree to which linear systems analysis could explain vestibular data. Some of these $[1,8,27]$ observed nonlinearities. Nonlinearities were found at high frequencies, where an amplitude dependence of gain arose because of cutoff of cell activity in the primary vestibular afferents [27]. In afoveate animals, nonlinearities have also been reported at lower frequencies. In the rabbit, phase lead and gain of the vestibular response was found to be mildly dependent on stimulus amplitude [1,8,12]. However, this amplitude dependence was not as pronounced in the rabbit as it was in the mouse. The present results suggest that the amplitude dependence of gain and phase arises because of the strong acceleration dependence of both quantities.

It is conceivable that the amplitude dependence of the VOR is partly caused by the presence of a scleral search coil. A possible way in which amplitude dependent changes in gain could arise due to the coil, would be when a dead zone was present between movement of the eye and displacement of the coil. Such a dead zone would cause recorded eye movement to be smaller than actual eye movement and would thus slightly depress gain with respect to the actual performance of the animal. Recorded amplitude of the eye would always reflect the real amplitude of the eye minus the amplitude of the dead zone and thus the relative contribution of the dead zone would decrease as the real amplitude of the eye is increased. Therefore, depression of the gain would be more pronounced at lower stimulus amplitudes causing an amplitude dependence of the system. However, the effect of a dead zone in the coil recording would be the same under all stimulus conditions. Therefore any such amplitude dependence should also be present in the vVOR. Fig. 10 clearly shows this is not the case; gain of the vVOR is the same for both tested amplitudes.

Although backlash between coil and the eye did not occur, the amplitude dependence of the VOR may still be partly caused by a dead zone. This dead zone could be in the form of a threshold within the VOR circuitry. Such a threshold was found in the present experiments, since we were unable to elicit a VOR response for accelerations below $8 \% \mathrm{~s}^{2}$. However, it is possible that the response was simply undetectable within the combined noise of the mouse ocular motor system and our recording system. This explanation is supported by previous studies, which have argued against the presence of thresholds. In rabbits eye movements were recorded to vestibular stimuli with am- plitudes as small as $2.5 \mathrm{~min}$ arc at $0.22 \mathrm{~Hz}$ [35]; no apparent threshold could be detected.

\subsection{Relationship of current study to past recordings in the mouse}

There have been earlier attempts at recording eye movements in mice. The current study improves on these older investigations in a number of respects. GrüsserCornehls and Böhm [17] recorded eye movements using electro-oculography. The authors reported that the animals needed to be sedated to record compensatory eye movements and that responses could only be elicited in $50 \%$ of the tested animals. In contrast, we found robust optokinetic responses in all animals and sedation was never necessary. In addition, electro-oculography did not allow for accurate quantification of the eye movements since calibration was based upon the assumption that the gain of the OKR is unity at low stimulus velocities. Calibration was also a problem in a more recent mouse study using video oculography [20]. Katoh et al. [20] measured motion of the mouse pupil, and converted it to angular rotation of the eye based upon an assumption of an eye radius of $1.6 \mathrm{~mm}$. In calculating the distance of the pupil to the geometric center of the eye, they failed to deduct the depth of the anterior chamber (about $0.55 \mathrm{~mm}$ in the mouse). Finally, the present recordings also demonstrate the limitations of our own, previous investigations, using a larger search coil temporarily placed upon the eye [10,11,22]. Based on current recordings with the smaller, chronically implanted, subconjunctival minicoil, we now suspect that the larger coil, which surrounded the entire limbus of the eye, distorted the compensatory eye movements because it impeded the free motion of the eye. In comparison to previous results, optokinetic and vestibular gains were considerably higher in the current study. The VOR gain at $0.8 \mathrm{~Hz}-10^{\circ}$ amplitude was 0.43 when measured with a minicoil, compared to 0.25 using the larger coil $[10,11]$.

We have recently undertaken experiments to record eye movements using an improved video calibration strategy [33]. Although the use of a minicoil greatly improved on the results from previous coil recordings in mice [11,22], video recordings revealed that ocular motility was still slightly affected by coil placement. Gain in the VORL was marginally lower in animals that had been implanted with a search coil, compared to animals that had never been implanted. No effect of the coil on the phase of the eye movement with respect to head movement could be found [33]. Despite the effect the minicoil has on eye movement, there remain a number of advantages in using coil oculography. First, it is more difficult to precisely calibrate the video recordings. To calibrate the video, recorded pixel displacement needs to be converted into degrees of rotation. Any calculation of the angular displacement relies on an accurate measurement of the pupil radius. Second, contrary to coil recordings the video technique is subject to 
artifacts like blinks and loss of pupil detection due to interference from the illuminating light; therefore videotracking the eye continuously requires close attention. Maintaining a good quality video recording means, at least in some animals, occasionally adjusting the illumination and software tracking parameters. Thus, in neurophysiology studies where eye movement is only one of the variables being tracked, the more robust coil recording may be preferable.

\section{References}

[1] E. Baarsma, H. Collewijn, Vestibulo-ocular and optokinetic reactions to rotation and their interaction in the rabbit, J. Physiol. 238 (1974) 603-625.

[2] G.W. Balkema, N.J. Mangini, L.H. Pinto, J.W. Vanable Jr., Visually evoked eye movements in mouse mutants and inbred strains. A screening report, Invest. Ophthalmol. Vis. Sci. 25 (1984) 795-800.

[3] U.W. Buettner, U. Buttner, V. Henn, Transfer characteristics of neurons in vestibular nuclei of the alert monkey, J. Neurophysiol. 41 (1978) 1614-1628.

[4] S.C. Cannon, D.A. Robinson, Loss of the neural integrator of the oculomotor system from brain stem lesions in monkey, J. Neurophysiol. 57 (1987) 1383-1409.

[5] B. Cohen, V. Matsuo, T. Raphan, Quantitative analysis of the velocity characteristics of optokinetic nystagmus and optokinetic after-nystagmus, J. Physiol. (Lond.) 270 (1977) 321-344.

[6] H. Collewijn, Optokinetic eye movements in the rabbit: inputoutput relations, Vis. Res. 9 (1969) 117-132.

[7] H. Collewijn, The normal range of horizontal eye movements in the rabbit, Exp. Neurol. 28 (1970) 132-143.

[8] H. Collewijn, B.J. Winterson, J. van der Steen, Post-rotary nystagmus and optokinetic after-nystagmus in the rabbit linear rather than exponential decay, Exp. Brain Res. 40 (1980) 330-338.

[9] I.S. Curthoys, The response of primary horizontal semicircular canal neurons in the rat and guinea pig to angular acceleration, Exp. Brain Res. 47 (1982) 286-294.

[10] C.I. De Zeeuw, C. Hansel, F. Bian, S.K. Koekkoek, A.M. van Alphen, D.J. Linden, J. Oberdick, Expression of a protein kinase C inhibitor in Purkinje cells blocks cerebellar LTD and adaptation of the vestibulo-ocular reflex, Neuron 20 (1998) 495-508.

[11] C.I. De Zeeuw, A.M. van Alphen, S.K. Koekkoek, E. Buharin, M.P. Coesmans, M.M. Morpurgo, J. van den Burg, Recording eye movements in mice: a new approach to investigate the molecular basis of cerebellar control of motor learning and motor timing, Otolaryngol. Head Neck Surg. 119 (1998) 193-203.

[12] C.I. De Zeeuw, D.R. Wylie, J.S. Stahl, J.I. Simpson, Phase relations of Purkinje cells in the rabbit flocculus during compensatory eye movements, J. Neurophysiol. 74 (1995) 2051-2063.

[13] J.H. Fuller, Eye and head movements during vestibular stimulation in the alert rabbit, Brain Res. 205 (1981) 363-381.

[14] R. Gerlai, Gene-targeting studies of mammalian behavior: is it the mutation or the background genotype? (see comments) (published erratum appears in Trends Neurosci. 19 (1996) 271), Trends Neurosci. 19 (1996) 177-181.

[15] E. Godaux, G. Cheron, Testing the common neural integrator hypothesis at the level of the individual abducens motoneurones in the alert cat, J. Physiol. 469 (1993) 549-570.

[16] E. Godaux, C. Gobert, J. Halleux, Vestibuloocular reflex, optokinetic response, and their interactions in the alert cat, Exp. Neurol. 80 (1983) 42-54.

[17] U. Grusser-Cornehls, P. Bohm, Horizontal optokinetic ocular nystagmus in wildtype $(\mathrm{B} 6 \mathrm{CBA}+/+)$ and weaver mutant mice, Exp. Brain Res. 72 (1988) 29-36.

[18] B.J. Hess, W. Precht, A. Reber, L. Cazin, Horizontal optokinetic ocular nystagmus in the pigmented rat, Neuroscience 15 (1985) 97-107.

[19] G.M. Jones, K.E. Spells, A theoretical and comparative study of the functional dependence of the semicircular canal upon its physical dimensions, Proc. R. Soc Lond. 157 (1963) 403-419.

[20] A. Katoh, H. Kitazawa, S. Itohara, S. Nagao, Dynamic characteristics and adaptability of mouse vestibulo-ocular and optokinetic response eye movements and the role of the flocculo-olivary system revealed by chemical lesions, Proc. Natl. Acad. Sci. USA 95 (1998) 7705-7710.

[21] M.J. Keng, T.J. Anastasio, The horizontal optokinetic response of the goldfish, Brain Behav. Evol. 49 (1997) 214-229.

[22] S.K. Koekkoek, A.M. van Alphen, J. van den Burg, F. Grosveld, N. Galjart, C.I. De Zeeuw, Gain adaptation and phase dynamics of compensatory eye movements in mice, Genes Funct. 1 (1997) 175-190.

[23] C. Maioli, W. Precht, The horizontal optokinetic nystagmus in the cat, Exp. Brain Res. 55 (1984) 494-506.

[24] N.J. Mangini, J.W. Vanable Jr., M.A. Williams, L.H. Pinto, The optokinetic nystagmus and ocular pigmentation of hypopigmented mouse mutants, J. Comp. Neurol. 241 (1985) 191-209.

[25] J.C. Mitchiner, L.H. Pinto, J.W. Vanable Jr., Visually evoked eye movements in the mouse (Mus musculus), Vis. Res. 16 (1976) $1169-1171$.

[26] S. Nagao, Effects of vestibulocerebellar lesions upon dynamic characteristics and adaptation of vestibulo-ocular and optokinetic responses in pigmented rabbits, Exp. Brain Res. 53 (1983) 36-46.

[27] G.D. Paige, Vestibuloocular reflex and its interactions with visual following mechanisms in the squirrel monkey. I. Response characteristics in normal animals, J. Neurophysiol. 49 (1983) 134-151.

[28] T. Raphan, V. Matsuo, B. Cohen, Velocity storage in the vestibuloocular reflex arc (VOR), Exp. Brain Res. 35 (1979) 229-248.

[29] D.A. Robinson, A method of measuring eye movement using a scleral search-coil in magnetic field, IEEE Trans. Biomed. Eng. 10 (1963) 137-145.

[30] D.A. Robinson, The effect of cerebellectomy on the cat's bestibuloocular integrator, Brain Res. 71 (1974) 195-207.

[31] D.A. Robinson, Adaptive gain control of vestibuloocular reflex by the cerebellum, J. Neurophysiol. 39 (1976) 954-969.

[32] A.A. Skavenski, D.A. Robinson, Role of abducens neurons in vestibuloocular reflex, J. Neurophysiol. 36 (1973) 724-738.

[33] J.S. Stahl, A.M. van Alphen, C.I. De Zeeuw, A comparison of video and magnetic search coil recordings of mouse eye movements, J. Neurosci. Methods 99 (2000) 101-110.

[34] A. Steinhausen, Uber die beobachtung der cupula in den bogengangsampullen des labyrinths der lebenden Hecht, Pflügers Arch. Ges. Physiol. 232 (1933) 500-512.

[35] B.J. Winterson, H. Collewijn, R.M. Steinman, Compensatory eye movements to miniature rotations in the rabbit: implications for retinal image stability, Vis. Res. 19 (1979) 1155-1159. 